\title{
The Rho-Associated Kinase Inhibitor Fasudil can Replace Y-27632 for Use in Human Pluripotent Stem Cell Research
}

Seongjun So ${ }^{1}$, Yeonmi Lee ${ }^{1}$, Jumi Park ${ }^{1}$, Ji-Yoon Lee ${ }^{2}$, Doin Kim ${ }^{1}$, Julie Hwang ${ }^{1}$, Joosung Shin ${ }^{1}$ Jiwan Choi ${ }^{1}$, Yuri Han ${ }^{1}$, Seoon Kang ${ }^{1}$, James R. Dutton ${ }^{3}$, Eul-Ju Seo ${ }^{4}$, Beom Hee Lee, Chong Jai Kim $^{5}$, Shoukhrat Mitalipov ${ }^{6}$, Soojin Oh ${ }^{2}$ \& Eunju Kang ${ }^{1,2, *}$

1 Stem Cell Center, Asan Institute for Life Sciences, Asan Medical Center, University of Ulsan College of Medicine, Seoul 05505, Republic of Korea; Soseongjun7@gmail.com (S.S.); yeonmilee82@gmail.com (Y.L.); jumipark1578@gmail.com (J.P.); doinclick@hanmail.net (D.K.); julie.hwang19@stu.siskorea.org (J.H.); joosung.shin20@stu.siskorea.org (J.S.); jiwanc624@gmail.com (J.C.); yuri664581@gmail.com (Y.H.); luceamso@gmail.com (S.K.)

2 Department of Convergence Medicine, Asan Institute for Life Sciences, Asan Medical Center, University of Ulsan College of Medicine, Seoul, 05505, Republic of Korea; ljy7901@amc.seoul.kr (J.Y.L.); diatree@amc.seoul.kr (S.J.O.)

3 Stem Cell Institute, University of Minnesota, Minneapolis, Minnesota, 55455, USA; dutto015@umn.edu (J.R.D.)

4 Medical Genetics Center, Asan Institute for Life Sciences, Asan Medical Center, University of Ulsan College of Medicine, Seoul, 05505, Republic of Korea; ejseo@amc.seoul.kr (E.J.S.); mdlbh@hanmail.net (B.H.L.)

5 Department of Pathology, Asan Institute for Life Sciences, Asan Medical Center, University of Ulsan College of Medicine, Seoul, 05505, Republic of Korea; ckim@amc.seoul.kr (C.J.K.)

6 Center for Embryonic Cell and Gene Therapy, Oregon Health \& Science University, Portland, Oregon, 97239, USA; mitalipo@ohsu.edu (S.M.)

* Corresponding: kangeun@amc.seoul.kr; Phone: +82-2-3010-8547; Fax: +82-2-3010-4182;

\begin{abstract}
Poor survival of human pluripotent stem cells (hPSCs) following freezing, thawing, or passaging hinders maintenance and differentiation in stem cell research. Rho-associated kinases (ROCKs) play a crucial role in hPSC survival. To date, a typical ROCK inhibitor, Y-27632, has been the primary agent used in hPSC research. Here, we report that another ROCK inhibitor, fasudil, can be used as an alternative. Fasudil increased hPSC growth due to survival rather than proliferation following thawing and passaging, similar to Y-27632. It did not affect pluripotency and genetic integrity including mitochondrial genome (mtDNA). Notably, the genes related to metabolism, mTORC1, and TP53 have mainly displayed a faster recovery pattern with ROCK inhibitors than control. Furthermore, fasudil was confirmed as useful for the single dissociation of hPSCs and for aggregation. It also increased retinal pigment epithelium (RPE) differentiation and the survival of neural crest cells during differentiation. These findings suggest that fasudil can replace Y-27632 for use in stem cell research.
\end{abstract}

Keywords: hPSCs; ROCK inhibitors; fasudil; cell survival; metabolism; differentiation

\section{Introduction}

Human pluripotent stem cells (hPSCs), such as embryonic stem cells (hESCs) or induced pluripotent stem cells (hiPSCs), are valuable research tools in the fields of developmental biology, disease modeling, drug screening, and regenerative medicine[1-3]. However, hPSCs are difficult to culture because of their poor survival, especially in circumstances of cellular detachment or dissociation for freezing and splitting[4-6]. Thus, hPSCs were originally cultured using the feeder layer technique to 
increase their survival and maintain pluripotency. Mouse embryonic fibroblasts (MEFs) are the most commonly used feeder cells in research, but MEFs may have limited utility for transplantation to humans due to xenogeneic contamination[5,7]. Another disadvantage of MEFs is that the use of any feeder layer, itself, leads to batch- or lab-dependent variations; additionally, feeder layers increase workload which subsequently limits large-scale hPSC culturing capacity[8,9]. To solve these problems, additional methods have been introduced, such as culturing hPSCs in suspension[10], with microcarriers[11], or on synthetic polymers[12]. As a result, feeder-free hPSC cultures became possible when hPSCs were grown on an extracellular matrix with specialized small molecules and growth factors[12,13].

In 2007, Y-27632 ([1R,4r]-4-[(R)-1-aminoethyl]-N-[pyridin-4-yl]cyclohexanecarboxamide), a small molecule that inhibits the Rho-associated kinase (ROCK) pathway, was reported to increase the survival and growth of PSCs; since then, Y-27632 has been used extensively in stem cell research [14]. The ROCK signal activates phospho-myosin light chain (pMLC), which contracts intracellular actomyosin and is known to induce apoptosis in dissociated cells. Y-27632 also has been used for PSC differentiation in retinal cells[15], endodermal lineage cells[16], insulin-producing cells[17], and for the maintenance of differentiated cells[18-20].

Recently, many researchers have used ROCK inhibitors as essential small molecules for PSC culture. Since Y-27632 was developed and put into use, researchers have primarily resorted to this ROCK inhibitor for PSC research. However, the synthesis of Y-27632 is achieved in seven steps, and, overall, its yield is only $45 \%[21]$. There are several ROCK inhibitors available on the market, including RKI1447, GSK429286A, H-1152, SLx-2119, TC-S 7001, and fasudil. In particular, fasudil (HA-1077, 5-[1,4diazepan-1-ylsulfonyl] isoquinoline) is synthesized only in one step, so it is much easier to synthesize than Y-27632[22]. To date, however, fasudil has only been used in PSC culture as a control group, but it has not been clearly characterized for use with hPSCs following long-term culture[14].

We compared the effectiveness of fasudil and Y-27632 in PSC culture in conditions of freezing, thawing, and splitting by long-term growth and maintenance of hPSCs under defined xeno-free conditions. We also investigated the effects of fasudil on the metabolism, the differentiation of PSCs into 3D aggregation, retinal pigment epithelium, and neural crest.

\section{Materials and Methods}

\subsection{Ethics and Governance}

For experiments involving human samples, informed consent had been obtained from participants and approved by the institutional review board (IRB: 2017-0260 and 2019-0117) at Asan Medical Center. Experiments on live vertebrates were performed in compliance with institutional regulations of the Asan Institute for Life Sciences and were approved Asan Institutional Animal Care and Use Committee (IACUC: 2018-12-269). All experiments were performed in accordance with relevant guidelines and regulations.

\subsection{Small Molecule Stability Analysis}

Briefly, samples (50 uL) were precipitated with $150 \mu \mathrm{L}$ of cold acetonitrile containing internal standard carbamazepine $(\mathrm{CBZ}, 10 \mathrm{ng} / \mathrm{mL})$ and agitated with a vortex mixer before centrifugation at $3400 \mathrm{rpm}$ and $4^{\circ} \mathrm{C}$ for 20 minutes. The supernatants were then analyzed by LC-ESI/MS/MS mass spectrometry with the Agilent 1200 series HPLC system (Agilent Technologies, Wilmington, DE, USA) and the API 4000 LC-MS/MS system equipped with a Turbo V IonSpray source (Applied Biosystems, Foster City, CA, USA) operated in the positive ion mode. Chromatographic separation was carried out on a Hypersil GOLD C18 column $(50 \times 4.6 \mathrm{~mm}$ i.d., $5 \mu \mathrm{m}$, Thermo Fisher Scientific, Waltham, MA, USA) for Y-27632, and on an Atlantis dC18 column (50×2.1 mm i.d., $3 \mu \mathrm{m}$, Waters, Milford, MA, USA) 
with a Security Guard C18 guard column $(2.0 \times 4.0 \mathrm{~mm}$ i.d., Phenomenex, Torrance, CA, USA) for fasudil. Detection was conducted using multiple reaction monitoring (MPM) of the transitions of $\mathrm{m} / \mathrm{z}$ 292>99 for fasudil, m/z 248>95 for Y-27632 and m/z 237>194 for CPZ (international standard). The retention times of fasudil and Y-27632 were 2.92 and 1.94 minutes, respectively. Acquisition and analysis of data were performed using Analyst software (ver. 1.5.2, Applied Biosystems). For all LCMS/MS analyses, the peak areas of the fasudil and Y-27632 were expressed as ratios to the internal standard (CPZ) peak area for each trial.

\subsection{Cell Culture}

Three hESC lines and four hiPSC lines were maintained based on the hPSC research guidelines of the Stem Cell Center, Asan Medical Center (Seoul, Republic of Korea). The hPSCs were cultured on vitronectin-coated $(5 \mu \mathrm{g} / \mathrm{ml}$; Life Technologies, Grand Island, NY) culture plates in Essential 8 medium (Life Technologies) at $37^{\circ} \mathrm{C}, 5 \% \mathrm{CO}^{2}[23,24]$. The cultures were passaged every week in clusters by chemically splitting the PSC using $1 \mathrm{mM}$ sodium citrate (S4641, Sigma-Aldrich, St. Louis, MO) or Gentle Cell Dissociation Reagent (Stem Cell Technologies, Vancouver, BC, Canada). Splitting using sodium citrate was carried out as described previously[25], and Gentle Cell Dissociation Reagent was used to compare with sodium citrate according to the manufacturer's instructions. The hPSC clusters were transferred onto prepared vitronectin-coated culture plates supplemented with fasudil (10 uM; Adooq, Irvine, CA) or Y-27632 (10 uM; Sigma-Aldrich).

\subsection{Freezing and Thawing}

For freezing of hPSCs, $10 \mu \mathrm{M}$ of ROCK inhibitor (Y-27632 or fasudil) was used for pre-treatment in culture medium for 30 minutes before freezing. hPSCs were collected in small clumps with sodium citrate, centrifuged (300 $g$ for 1 minute) and resuspended with Essential 8 medium. Cell suspensions were slowly mixed with freezing medium (20\% DMSO in culture medium) in a final volume ratio of 1:1[26]. The cells mixed with freezing medium were dispensed into cryovials (Life Technologies), which were immediately placed into CoolCell LX Freezing Containers (Sigma-Aldrich). The vials were stored overnight in an $-80^{\circ} \mathrm{C}$ freezer to provide an approximately $1^{\circ} \mathrm{C} /$ minute cooling rate. The following day, the vials were placed in either $\mathrm{LN} 2$ or at $-80^{\circ} \mathrm{C}$ for storage. For thawing, cryovials were rapidly warmed in a $37^{\circ} \mathrm{C}$ water bath for approximately 1 minute until the ice disappeared. The cell suspension was then transferred to a $15 \mathrm{ml}$ centrifuge tube and slowly mixed with $5 \mathrm{ml}$ of warm culture medium. After centrifugation (300 $g$ for 1 minute), the supernatant solution was removed, and cell pellets were resuspended with $1 \mathrm{ml}$ of fresh culture medium. The cells were plated in vitronectin-coated 6-well plates and cultured in the conditions described above.

\subsection{Alkaline Phosphatase Activity Analysis}

The Alkaline Phosphatase Substrate Kit I (System Biosciences, Palo Alto, CA) was used to analyze alkaline phosphatase activity within clusters according to the manufacturer's instructions.

\subsection{Cell Counting}

To examine cell growth, sub-confluent cells were dissociated to clumps using sodium citrate or Gentle Cell Dissociation Reagent. The part of the solution containing cell clumps was dissociated into single cells with $0.25 \%$ trypsin-EDTA for cell counting.

\subsection{Cell Proliferation Analysis}

To determine proliferation, on the third day after the split, hPSCs were collected and fixed with $10 \%$ neutral buffered formalin (BBC Biochemical, Mount Vernon, WA). Cells were permeabilized with $0.05 \%$ Triton X-100 in $0.01 \mathrm{M}$ sodium citrate for 30 minutes at room temperature. Fixed cells were washed twice with phosphate-buffered saline (PBS, Hyclone, Chicago, IL) and permeabilized with 
0.05\% Triton X-100 in $0.01 \mathrm{M}$ sodium citrate for 30 minutes at room temperature. Ki-67 (Abcam, Cambridge, UK) primary antibody were diluted at 1:200 in PBS containing 10\% fetal bovine serum (FBS, Life Technologies) and incubated overnight in the refrigerator. After the incubation, cells were washed three times with PBS and incubated for 1 hour in the refrigerator with Goat Anti-Rabbit IgG H\&L (1:500, Alexa Fluor 555, Abcam). Samples were counted using FACS (Canto II, BD Biosciences). The FACS data was analyzed using FlowJo xV.0.7 software (Tree Star, Ashland, OR).

\subsection{Quantitative Polymerase Chain Reaction ( $q P C R$ )}

The total RNA of hPSCs cultured in the presence of fasudil or Y-27632 was isolated using RNeasy Mini Kits (Qiagen, Valencia, CA), and reverse transcription was performed with cDNA synthesis kits (PCR Biosystems, London, UK) according to the manufacturers' instructions. The results were further confirmed using conventional real-time polymerase chain reaction (PCR) analysis[27]. Reverse transcriptase quantitative PCR (RT-qPCR) was then performed using each gene-specific primer, Power SYBR Green PCR Master Mix, and the QuantStudio 6 Flex Real-Time PCR System (Applied Biosystems, Foster City, CA) according to the manufacturer's protocol.

The primers used were: OCT4 F-5'-GACAGGGGGAGGGGAGGAGCTAGG-3', R-5'CTTCCCTCCAACCAGTTGCCCCAAA-3', SOX2 F-5'-AGCTACAGCATGATGCAGGA - $3^{\prime}{ }^{\prime}$, R-5'GGTCATGGAGTTGTACTGCA-3', NANOG F-5'-TGAACCTCAGCTACAAACAG-3', R-5'TGGTGGTAGGAAGAGTAAAG-3', ROCK1 F-5'-TACCAGCTGCAAGCTATATTAGA-3', R-5'TTCGAGATTATGTTTGAGATGC-3', ROCK2 F-5'-ATCAAAGAGATGATGGCTAGAC-3', R-5'GCAAGATTG GCAACATCAC-3', PKA F-5'-TGACGTCAGTAGCCGAACGC-3', R-5'CCAGACTCCATG GTTCTCTC-3', PKC F-5'-CACGAGGTGAAGGACCACAA-3', R-5'TGACGCATTGCT TGTGAACG-3', PKG F-5'-GTGTGATGGAGTGGGACCAG-3', R-5'CGAGTGAATGGGCGCTAAGT-3', CASP3 F-5'-AATTGTGGAATTGATGCGTGATG-3', R-5'CTACAACGATCCCCTCTGAAAAA-3'[28], mTORC1 F-5'-CTGGGACTCAAATGTGTGCAGTTC-3', R-5'-GAACAATAGGGTGAATGATCCGGG-3'[29], TP53 F-5'-CCAGGGCAGCTACGGTTTC-3', R5'-CTCCGTCATGTGCTGTGACTG-3'[30]. The qPCR was performed in a final volume of $20 \mu \mathrm{l}$ containing $10 \mu \mathrm{l}$ SYBR Green master mix, $2 \mu \mathrm{l}$ cDNA (50 $\mathrm{ng} / \mu \mathrm{l}), 0.5 \mu \mathrm{l}$ each forward and reverse primer $(10 \mathrm{pmol})$ and $7 \mu \mathrm{l}$ nuclease-free water and the annealing temperature was $62 \sim 65^{\circ} \mathrm{C}$. The PCR data was analyzed and normalized with GAPDH expression with QuantStudio 6 and 7 Flex Software (Applied Biosystems).

\subsection{Immunocytochemistry}

Fasudil-treated hPSCs were seeded onto vitronectin-coated 24-well plates. Five days after plating, hPSCs were fixed in $10 \%$ neutral buffered formalin overnight in a refrigerator $\left(2-8^{\circ} \mathrm{C}\right)$, washed twice with PBS and, if required, permeabilized with $0.05 \%$ Triton X-100 in $0.01 \mathrm{M}$ sodium citrate for 30 minutes at room temperature. Primary antibodies were diluted at 1:100 to 1:500 in PBS containing 10\% FBS and incubated overnight in the refrigerator. The primary antibodies, TRA-1-60, SSEA4, and Oct4 (1:100, Stemgent, Cambridge, MA), were used for pluripotency analysis, and PAX3, PAX6, SOX10, and MITF (1:200, Abcam) were used for differentiation analysis. After the incubation of primary antibodies, cells were washed three times with PBS and incubated for 1 hour at room temperature with secondary antibodies. The secondary antibodies were Goat Anti-Mouse IgG H\&L (1:1000, Alexa Fluor 488, Abcam), Goat Anti-Rabbit IgG H\&L (1:500, Alexa Fluor 555, Abcam) and Goat Anti-Rabbit IgG H\&L (1:200, Alexa Fluor 647, Abcam). Samples were imaged and captured using a Carl Zeiss inverted microscope with an AxioCam MRc Rev 3 digital camera (Zeiss, Oberkochen, Germany; Confocal Imaging Core Laboratory, ASAN Institute for Life Sciences, Seoul, Republic of Korea).

\subsection{Teratoma Assay}


Teratoma formation experiments were performed by injecting hPSCs (maintained in the presence of fasudil for 3 months and detached from vitronectin-coated plates as cell clumps containing fasudil) into the femoral region of 7-week-old NOD-SCID Gamma mice (NSG, The Jackson Laboratory, Bar Harbor, ME) using a $1 \mathrm{ml}$ syringe (Korea Vaccine Co., Seoul, Republic of Korea) [31]. Mice with tumors were euthanized, and teratomas were isolated, sectioned, and histologically characterized for the presence of representative tissues with hematoxylin and eosin (H\&E)[32].

\subsection{Karyotyping}

Karyotyping was carried out on hPSC lines (hES1, hES2, hES3, hiPS1, hiPS2, and hiPS 3-C4) maintained in the presence of $10 \mu \mathrm{M}$ fasudil over 3 months by standard $\mathrm{G}$ banding at the Medical Genetic Center, Asan Medical Center (Seoul, Republic of Korea)[33].

\subsection{Single Cell Colony Forming Assay}

Accutase (StemCell Technologies) was used to dissociated hPSCs into single cells using, which were then plated at $5 \times 10^{4}$ cells/well on 6-well plates coated with vitronectin in Essential 8 medium. The medium was changed every day.

\subsection{D Serum-Free PSC Aggregation}

The culturing of aggregated cells was carried out as described previously with a minor modification[14]. Dissociated hPSCs (prepared as above; $1.2 \times 10^{6}$ cells $/ \mathrm{ml}$ ) were seeded onto a StemFIT 3D culture dish (MicroFIT, Republic of Korea) and cultured in DMEM/F12 (Life Technologies) supplemented with 20\% Knockout Serum Replacement (KSR, Life Technologies), 2 mM glutamine (Life Technologies), $0.1 \mathrm{mM}$ nonessential amino acids (Life Technologies), $0.1 \mathrm{mM} 2$ ME (Sigma-Aldrich), $100 \mathrm{U} / \mathrm{ml}$ penicillin, and $100 \mathrm{mg} / \mathrm{ml}$ streptomycin (GE Life Science, Chicago, IL). Then, $10 \mu \mathrm{M}$ of ROCK inhibitor (Y-27632 or fasudil) was added to the culture medium for the first 24 hours, and dissociated hPSCs were cultured for 6 days in StemFIT 3D culture dishes.

\subsection{Differentiation}

Retinal pigment epithelium (RPE) cells were derived by in vitro differentiation of hPSCs as previously described[34]. Briefly, hPSCs were plated at $70 \%$ to $80 \%$ confluency on 24 -well plates coated with vitronectin in basal neural induction medium (DMEM/F12 supplemented with N2B27, and NEAA, Life Technologies). After plating the cells, differentiation was performed for 2 days in basal neural induction medium supplemented with nicotinamide (10 mM, Sigma), noggin ( $50 \mathrm{ng} / \mathrm{ml}$, Peprotech, Rocky Hill, NJ), DKK (10 ng/ml, Peprotech), and IGF1 (10 ng/ml, Peprotech). The following 2 days, FGF2 (5 ng/ml, Peprotech) was added to the medium as the preceding differentiation medium. Subsequently, cells were cultured with basal neural induction medium supplemented with activin A (100 ng/ml, Peprotech) for 2 days. For the next 7 days, the medium was switched to basal neural induction medium supplemented with activin A (100 ng/ml, Peprotech), CHIR99021 (3 $\mu \mathrm{M}$, Peprotech), and SU5402 (10 $\mu \mathrm{M}$, Peprotech). The differentiation process also included ROCK inhibitor (10 $\mu$ M, Y-27632 or Fasudil) treatment; the medium was replaced every day.

Differentiation of neural crest cells was carried out according to previously described procedures with minor modifications[33,35]. We used combinations of small molecule activators of WNT and FGF2 signaling and inhibitors of ALK signaling to achieve directed neural crest differentiation from hPSCs. ROCK inhibitors (10 $\mu \mathrm{M}, \mathrm{Y}-27632$ or Fasudil) were also treated in the differentiation process.

\subsection{Necrotic and Apoptotic Cell Analysis}

Apoptotic analysis of hPSCs was carried out using the Dead Cell Apoptosis Kit with Annexin V Alexa Fluor 488 \& Propidium Iodide (Invitrogen). Staining with Annexin V/PI was carried out on floating 
and attached cells as follows. Floating cells in the medium were collected and retained. Annexin V/PIstained cells were further counted using FACS (Canto II, BD Biosciences)

\subsection{Statistical Analysis}

Statistical comparisons between the two groups were made using Student's two-tailed t-test or twotailed paired t-test. One-way ANOVA with Tukey post hoc analysis using Prism 5 software (GraphPad, San Diego, CA) was applied when more than two groups were compared. Data are expressed as mean \pm standard deviation (SD) or \pm standard error of the mean (SEM).

\section{Results}

\subsection{Comparison of Fasudil and Y-27632}

We searched for a method to increase cell survival during PSC culture and selected fasudil as a candidate small molecule. Fasudil has only been used in comparison with Y-27632 [14, 27, 28] or partially for differentiation studies[36]. Fasudil is a ROCK2 inhibitor with molecular differences from Y-27632 (Figure 1A)[27,37]. This small molecule also inhibits the protein kinase group AGC (PKA, PKG, and PKC), which are known to regulate the self-renewal and survival of hPSC[38,39]. While Y27362 retains a high price due to its complicated synthesis process, fasudil is relatively simple to synthesize and about 9.8 to 29.8 times lower in price than Y-27632 (Figure 1B)[21,22].

In the above study, we hypothesized that fasudil-treat media need not be reprocessed into fasudil to produce new media after prolonged storage $\left(2-8^{\circ} \mathrm{C}\right)$. In addition, it was assumed that the fasudil could remain in the medium for a long time without changing the medium for 2 days or more in the process of PSC culture or differentiation. To determine how long fasudil could remain, we investigated the stability of fasudil in refrigerated media or in hPSC culture conditions and compared the results to those of Y-27632 (Figure 1C). Fasudil with complete culture medium was stable for 6 days after being stored in the refrigerator. Y-27632 was also stable during 6 days. Both Y-27632 and fasudil were stable for up to 2 days in PSC culture $\left(37^{\circ} \mathrm{C}, 5 \% \mathrm{CO}_{2}\right)$.

Fasudil inhibits $0.33 \mu \mathrm{M} \mathrm{Ki}[40]$, Inhibition Constant (Ki) which the rate constants for the binding of the inhibitor to the enzyme, and also PKA, PKC, and PKG with $\mathrm{Ki}$ of $1.6,3.3$ and $1.6 \mu \mathrm{M}$, respectively[41]. Generally, the inhibitor concentrations of substrates are recommended at or above $3 \sim 10$ times $\mathrm{Ki}$ to ensure maximum inhibition and the identification of any potential partial inhibitors[42]. To determine the suitable amount of the molecule for the PSC maintenance, we optimized the fasudil concentration based on mRNA expressions of ROCK1, ROCK2, PKA, PKC, and PKG in hES3 and hiPS2 cells treated with 1.6, 3.3, and $10 \mu \mathrm{M}$ of fasudil. All of the gene expressions were similar among the groups (Figure 1D). The PKG gene tended to gradually decrease as the concentration increased. We also tested whether fasudil was effective in certain concentrations for PSC survival. Randomly selected hESCs had significantly higher growth in concentrations over $1 \mu \mathrm{M}$ than in untreated the control medium. The growth of hESCs cultured at $5 \mu \mathrm{M}$ and $10 \mu \mathrm{M}$ was found to be similar without any PSC morphological changes (Figure 1E). Finally, we compared the ratio of the expression between ROCK1 and ROCK2 genes at $10 \mu \mathrm{M}$. The ROCK2 expression was about two times greater than ROCK1 in four out of five lines of hPSCs (3 ESC lines and 2 iPSC lines, Figure 1F). 
A

\begin{tabular}{|c|c|c|}
\hline & Y-27632 & Fasudi \\
\hline $\begin{array}{c}\text { Chemical } \\
\text { Name }\end{array}$ & $\begin{array}{c}\text { (1R,4r)-4-((R)-1- } \\
\text { aminoethyl)-N-(pyridin-4- } \\
\text { yl)cyclohexanecarboxami } \\
\text { de }\end{array}$ & $\begin{array}{c}\text { HA-1077, } \\
\text { 5-(1,4-diazepan-1- } \\
\text { ylsulfonyl) isoquinoline }\end{array}$ \\
\hline $\begin{array}{c}\text { Molecular } \\
\text { Formula }\end{array}$ & $\mathrm{C}_{14} \mathrm{H}_{21} \mathrm{~N}_{3} \mathrm{O}$ & $\mathrm{C}_{14} \mathrm{H}_{17} \mathrm{~N}_{3} \mathrm{O}_{2} \mathrm{~S}$ \\
\hline $\begin{array}{c}\text { Molecular } \\
\text { Weight }\end{array}$ & $247.342 \mathrm{~g} / \mathrm{mol}$ & $291.369 \mathrm{~g} / \mathrm{mol}$ \\
\hline Structure & \begin{tabular}{c}
2 \\
\hline
\end{tabular} & \\
\hline
\end{tabular}

B

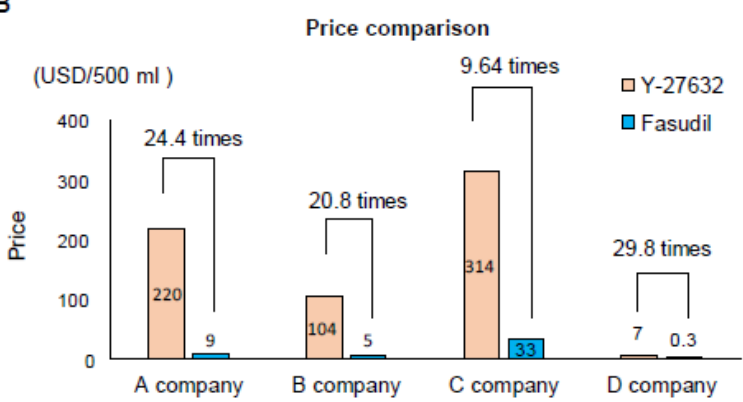

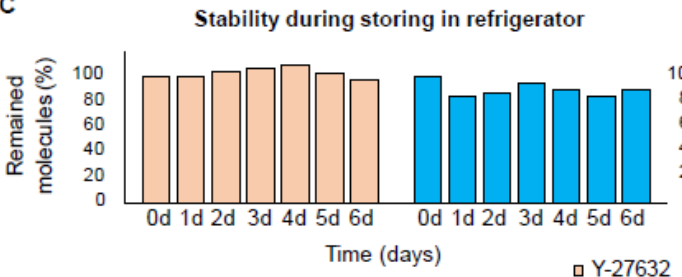

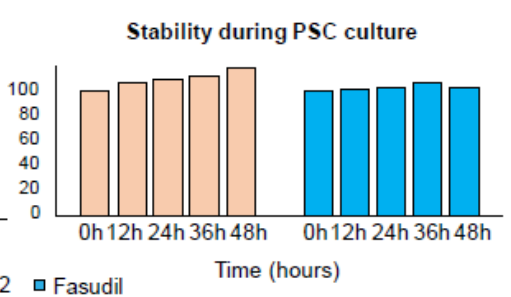

$\mathbf{E}$

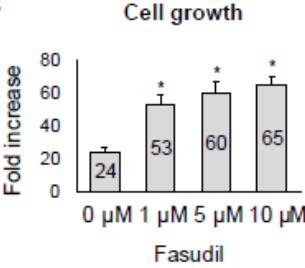

D

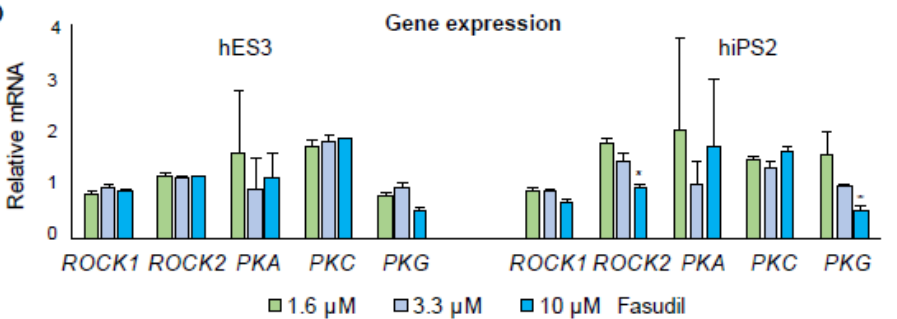

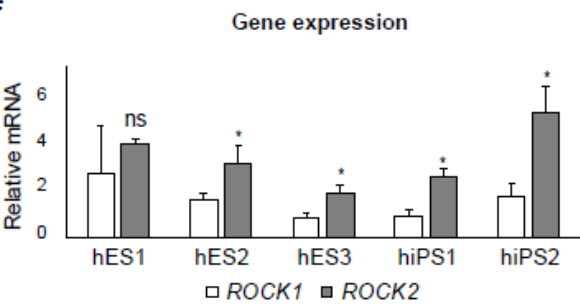

Figure 1. Molecular characteristics of fasudil and Y-27632. (A) Characteristics of Y-27632 and fasudil. Y-27632 has a structure that is difficult to synthesize in comparison to fasudil. (B) Price comparison of Y-27632 and fasudil. Y-27632 is 10 to 30 times more expensive than fasudil depending on the sales company. (C) Comparison of fasudil and Y-27632 stability over time while refrigerated or during PSC culture. The stability of fasudil was maintained similar to that of Y-27632 when stored in the refrigerator and cultured in the incubator $\left(5 \% \mathrm{CO}_{2}, 37^{\circ} \mathrm{C}\right)$. (D) Quantitative RT-PCR analysis to determine ROCK1, ROCK2, PKA, PKC, and PKG gene expressions relative to GAPDH of hES3 and hiPS2 cells cultured with 1.6, 3.3, and $10 \mu \mathrm{M}$ of fasudil. When fasudil was treated, the gene expression of PKG was decreased as the concentration increased, but expressions of the other genes were similar at all concentrations. Statistical significance ( $p$-value) was calculated using one-way ANOVA with Tukey post hoc analysis, ${ }^{*} p<0.05 ; \mathrm{n}=6$, Error bars indicate standard deviations. (E) Growth of hES3 cells for 7 days according to fasudil concentration. The growth of hES3 cells at fasudil concentrations of 1,5 , and $10 \mu \mathrm{M}$ was not significantly different $\left(^{*}: p<0.05 ; \mathrm{n}=3\right)$. (F) Quantitative RT-PCR analysis determining ROCK1 and ROCK2 gene expression relative to GAPDH $\left(^{*}: p<0.05 ; \mathrm{n}=9\right)$. In four out of five PSC lines, ROCK2 expression was significantly higher than ROCK1 expression. Statistical significance (p-value) was calculated using Student's t-test.

In summary, fasudil was a stable small molecule within a culture medium in a refrigerator or incubator and had broad effective concentrations. Therefore, we determined the working concentration at $10 \mu \mathrm{M}$ in the followed studies.

\subsection{Comparable Growth of PSCs Supplemented with Fasudil and Y-27632}

Generally, ROCK inhibitors are used to prevent cell death caused by the freezing and thawing of hPSCs. To determine whether fasudil has a similar effect as Y-27632, we compared cell survival after the freezing and thawing of randomly selected hESC and hiPSC lines. After freezing and thawing, we cultured hPSCs for 7 days and then measured cell survival by the fold increase of cell number 
(fold increase; cell number after 7 days culture/seeded cell number). The hPSCs (hES2 and hiPS1) supplemented with Y-27632 or fasudil grew much faster than the non-treated controls without morphological changes (Figure $2 \mathrm{~A}$ and $\mathrm{B}, p<0.05$ ). Surprisingly, in hESCs, the fasudil-treated group had a significantly higher growth rate than the group using Y-27632 (8.1 vs. 6.7, $p<0.05)$. In the case of hiPS1, there was no difference between the fasudil- and Y-27632-treated groups (8.4 vs. 8.3).

Next, we compared fasudil and Y-27632-mediated hPSC growth in five lines of hPSCs under feederfree and defined culture conditions. Passaging was proceeded by an enzyme-free method, which was part of a commercial or laboratory protocol[25]. We evaluated cell growth by the fold increase of cell number and the undifferentiated state by colonial morphology and alkaline phosphatase (ALP) positivity. When hPSCs were dissociated and seeded without a ROCK inhibitor, the fold increases were 4 to 39 times whereas, in the fasudil-supplemented culture, the fold increases were 10 to 89 times depending on the cell line, similar to Y-27632 culture (Figure 2C and D). hES1, which was one of the hESC lines that showed no difference between the groups, indicating a lack of effect of the ROCK inhibitors. ALP staining was carried out in cultured hPSCs (hES3 and hiPS2) with or without ROCK inhibitors. hPSC colony formation was similar between fasudil and Y-27632, while both treated groups exhibited higher rates of colony formation than the untreated control (Figure 2E). Furthermore, we measured the accumulation of cell growth during three passages. The total number of cells increased by about 100,000-fold through three passages, which was similar to the group treated with Y-27362 (about 90,000-fold). However, in the untreated group, the number of cells increased only about 2,400-fold (Figure 2F).
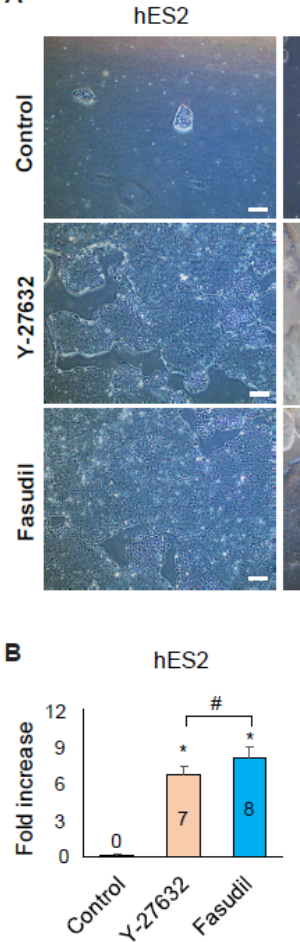

hiPS1
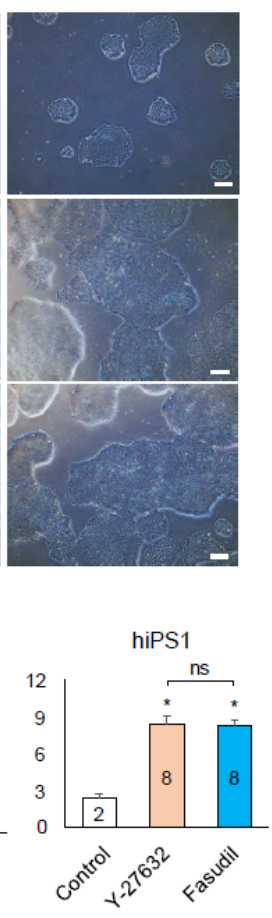

c

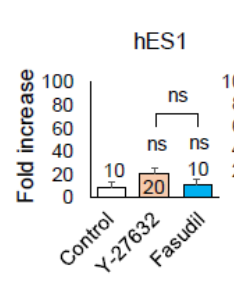

D
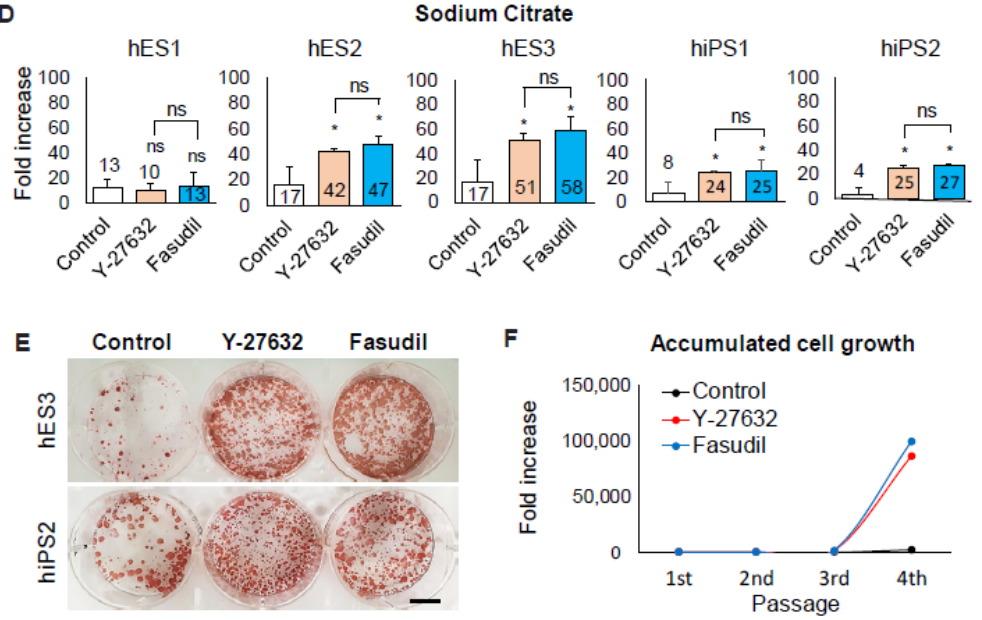

Gentle Cell Dissociation Reagent

hES2
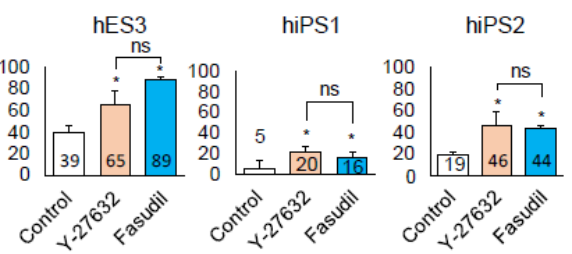

Sodium Citrate

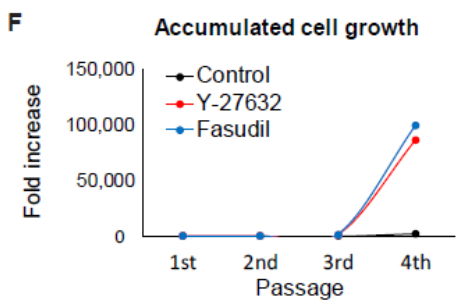

Figure 2. Fasudil enhances cell survival and the proliferation of hPSCs. (A) Typical colony morphologies of hPSCs (hES2 and hiPS1) cultured using Y-27632 or fasudil for 7 days after thawing. When treated with fasudil, the colony was larger than the control group. Scale bars $=200 \mu \mathrm{m}$. (B) Fold increase of Y-27632- or fasudil-supplemented culture for 7 days after thawing. There was no difference between fasudil and Y-27632 treatment ( ${ }^{*}: p<0.05$; versus control, $\mathrm{n}=6$ ). (C,D) Fold increase of the number of initially seeded hESCs (hES1, 2, and 3) and hiPSCs (hiPS1 and 2) using Gentle Cell Dissociation Reagent (C) and sodium citrate (D) Cell growth increased when fasudil was treated on all hPSC lines except hES1, and its effect was similar to that of Y-27632 $\left(^{*}: p<0.05\right.$; versus control, $n=3$ ). Statistical significance ( $p$-value) of results was calculated using one-way ANOVA with 
Tukey post hoc analysis. Error bars indicate standard deviations. (E) ALP staining of hES3 and hiPS2 cells cultured with Y-27632 or fasudil. When treated with fasudil, the PSC colony was larger than the control group, and the effect was similar to that of Y-27362 treatment. Scale bar $=10 \mathrm{~mm}$. (F) Accumulation of the cell count during three passages. After three passages, treatment with fasudil and Y-27632 resulted in a dramatic increase in cell number when compared to the untreated group.

To understand the mechanisms of PSC growth, we first measured cell death rates using Annexin V and PI. The hES3 and hiPS2 were seeded, then necrosis and apoptosis were examined 24 hours later. The majority of the seeded cells $(43 \sim 70 \%)$ had disappeared with significantly higher non-treated controls (Figure 3A, $p<0.05$ ). Both the fasudil and Y-27632 treated groups had lower necrotic rates than the non-treated controls ( 26 or $27 \%$ vs. $62 \%$ at hES3, 27 or $30 \%$ vs. $44 \%$ at hiPS2, $p<0.05$ ), while they had comparable apoptotic rates (Figure 3B). Furthermore, we investigated the mRNA expression of the apoptotic gene, CASP3. The samples were collected at $0,12,24,48$, and 96 hours after seeding the PSCs. In general, the expression of CASP3 decreased in all groups at 24 hours and 96 hours, similarly to the previous report[43]. hES3 was expressed differently at 12, 48 and 96 hours for nontreated controls (Figure 3C, $p<0.05$ ). Secondly, we performed a proliferation assay with the Ki-67 protein expression. Both of the small molecules did not affect cell proliferation (Figure 3D).

We finally investigated the dynamics of gene expressions closely related to metabolic activity, mTORC1 and TP53 [44-47], which has been reported to be a similar pattern of metabolites in hPSC [43]. The samples (non-treated control, Y-27632 or fasudil treated) were collected at 0, 12, 24, 48, and 96 hours after seeding the hPSCs on the plates in hES3 and hiPS2. The expressions of $m$ TORC1 rapidly decreased by 24 hours and gradually recovered to the next passaging (Figure 3E). Notably, the treated groups displayed significantly faster restore patterns than the control group. The expression dynamics of TP53 was similar to mTORC1. 

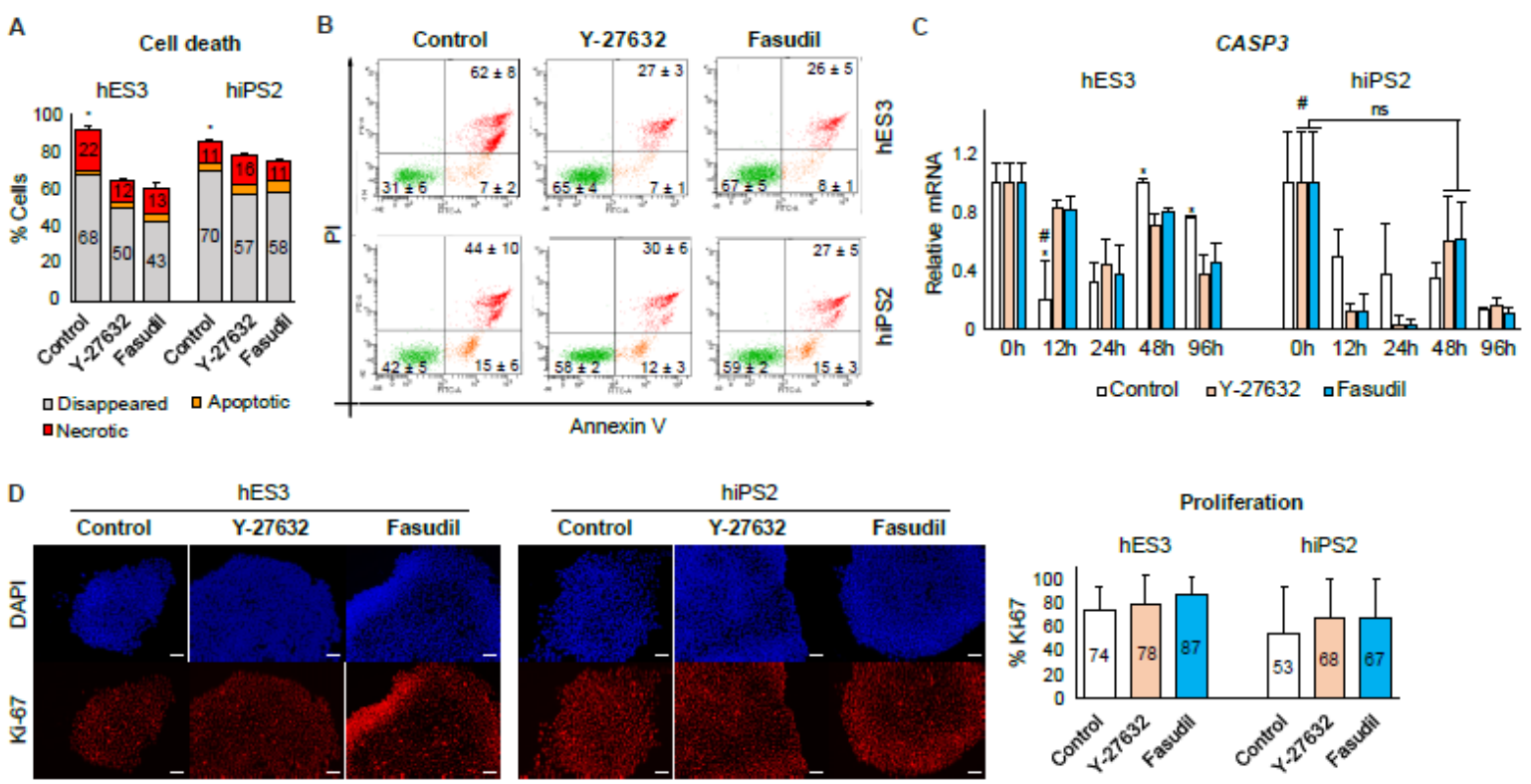

$\mathrm{E}$
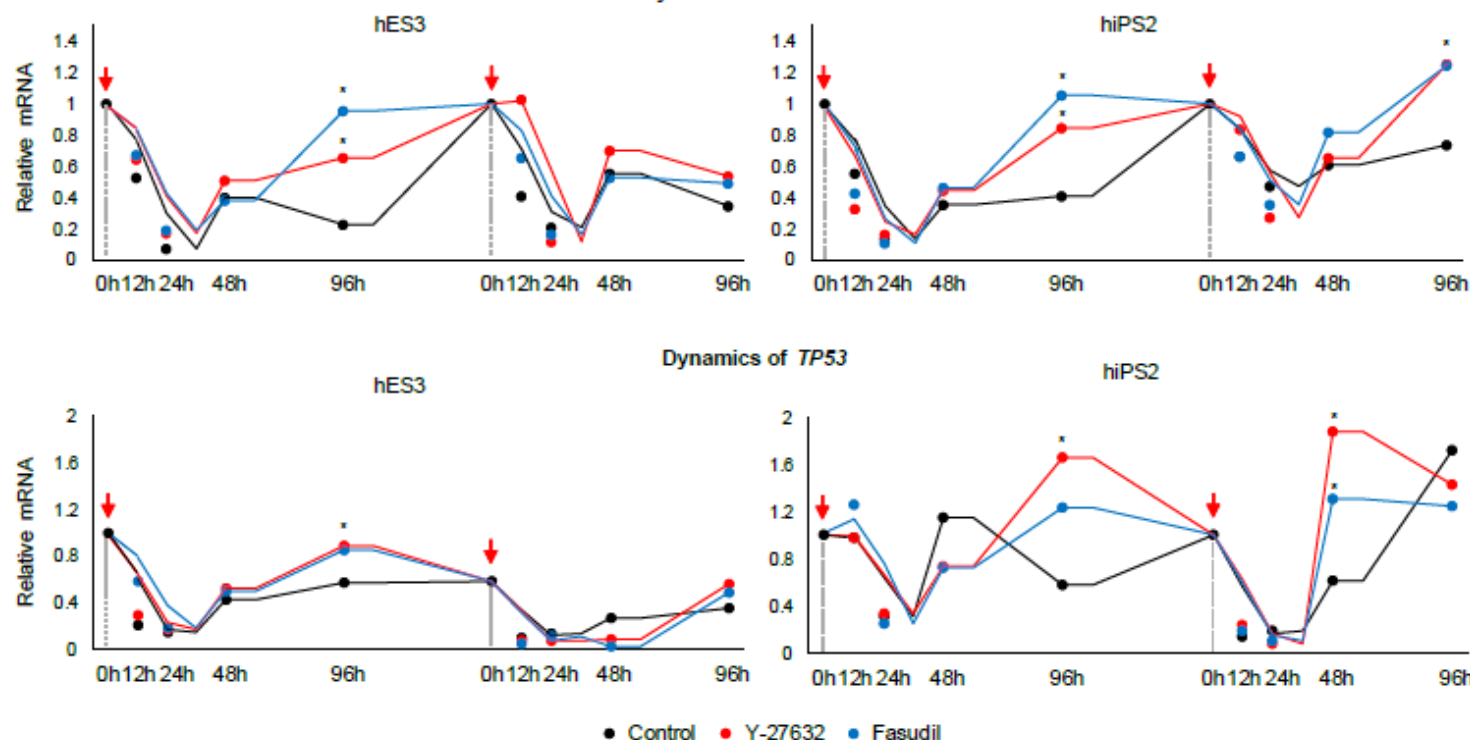

Figure 3. The mechanism of PSC growth. (A) The total cell death rate (\%) 24 hours after 100,000 cells were plated on 6 well plates (Disappeared $=100,000$ - Number of cells collected 24 hours later, $\%=$ [100,000 - disappeared cells] x Necrotic or apoptotic percentage/100). The total cell death was significantly higher in the control group. (B) Analysis of live, necrotic, and apoptotic cell ratio using annexin V/PI staining of hES3 and hiPS2 cells 24 hours after seeding. Compared with the control group, the necrotic cells had decreased when treated with fasudil ( $* p<0.05 ; n=3)$. (C) Quantitative RT-PCR analysis of CASP3 gene expression relative to GAPDH of hES3 and hiPS2 cells (*:significance between groups within each time, \#: significance over time in each group, $p<0.05 ; n=6)$. (D) Cell proliferation analysis using immunofluorescence (left) and flow cytometry (right) to examine Ki-67 expression of hES3 and hiPS2. There were no significant differences among the groups (versus control, $n=3$ ). Scale bar $=100 \mu \mathrm{m}$. (E) Quantitative RT-PCR analysis to determine mTORC1 and TP53 gene expression relative to GAPDH of hES3 and hiPS2 cells (Red arrow: pasaging points). In all experimental groups, the mTORC1 expression decreased rapidly after the cells were seeded on the plates, being lowest at 24 hours and then gradually recovered. The recovery rate was faster when treated with ROCK inhibitors ( ${ }^{*}: p<0.05 ; \mathrm{n}=3$ ). TP53 expression pattern was similar to mTORC1. Statistical significance ( $\mathrm{p}$-value) of the results were calculated using one-way ANOVA with Tukey 
post hoc analysis. Statistical significance (p-value) of the results were calculated using one-way ANOVA with Tukey post hoc analysis. Error bars indicate standard deviations.

In conclusion, fasudil not only promoted the growth of hPSCs through the enhancement of cell survival following thawing and splitting, but also helps to restore the metabolism quickly, and the efficacy was similar or better than Y-27632,.

\subsection{Maintaining Pluripotency Following Long-Term Fasudil Treatment}

To confirm that fasudil does not induce a change of pluripotency following treatment, five hPSC lines were sub-cultured with $10 \mathrm{uM}$ of fasudil over 2 to 3 months and were characterized to demonstrate their pluripotent characteristics. The represented PSC markers, including OCT4, SOX2, NANOG, SSEA4, and TRA-1-60, were analyzed in the PSCs by qPCR and immunocytochemistry (ICC)[48]. The gene expression levels, including OCT4, NANOG, and SOX2, were not significantly different among the treatment groups, while individual PSC lines were expressed differently (Figure 4A). OCT4, SSEA4, and TRA-1-60 proteins were detected in all of the cell lines (Figure 4B). Additionally, the karyotypes showed normal chromosome arrangements suggesting stable genome integrity (Figure 4C). Finally, we injected five cell lines into immunosuppressed mice (NSG, The Jackson Laboratory, Bar Harbor ME) to confirm teratoma formation. All cell lines generated teratomas with three germ layers-ectoderm, mesoderm, and endoderm (Figure 4D). These findings demonstrated that fasudil does not inhibit the pluripotency or genetic integrity of PSCs. 

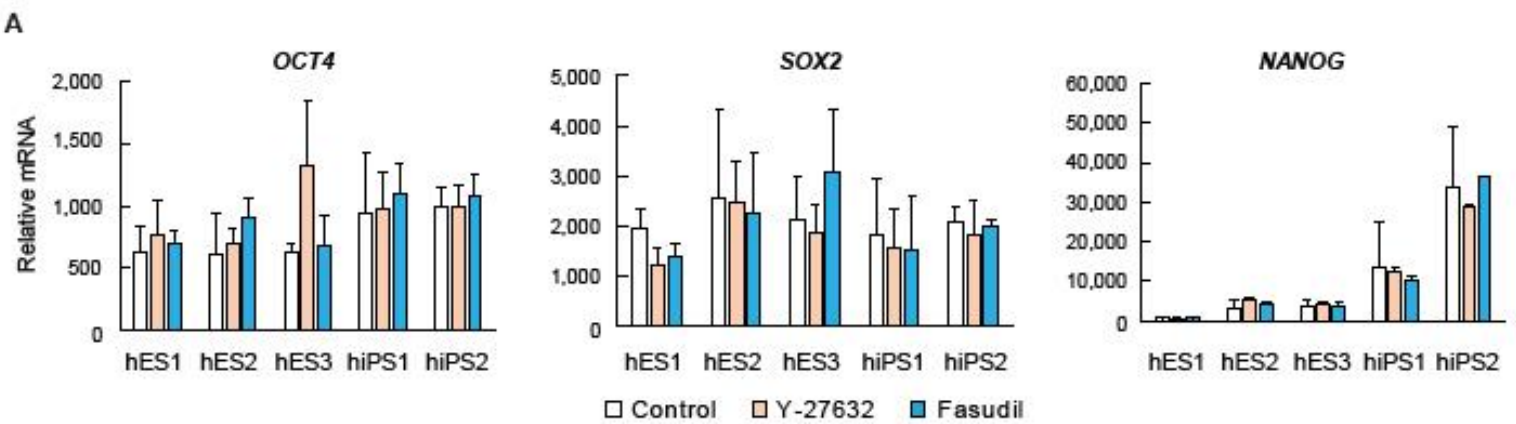

B
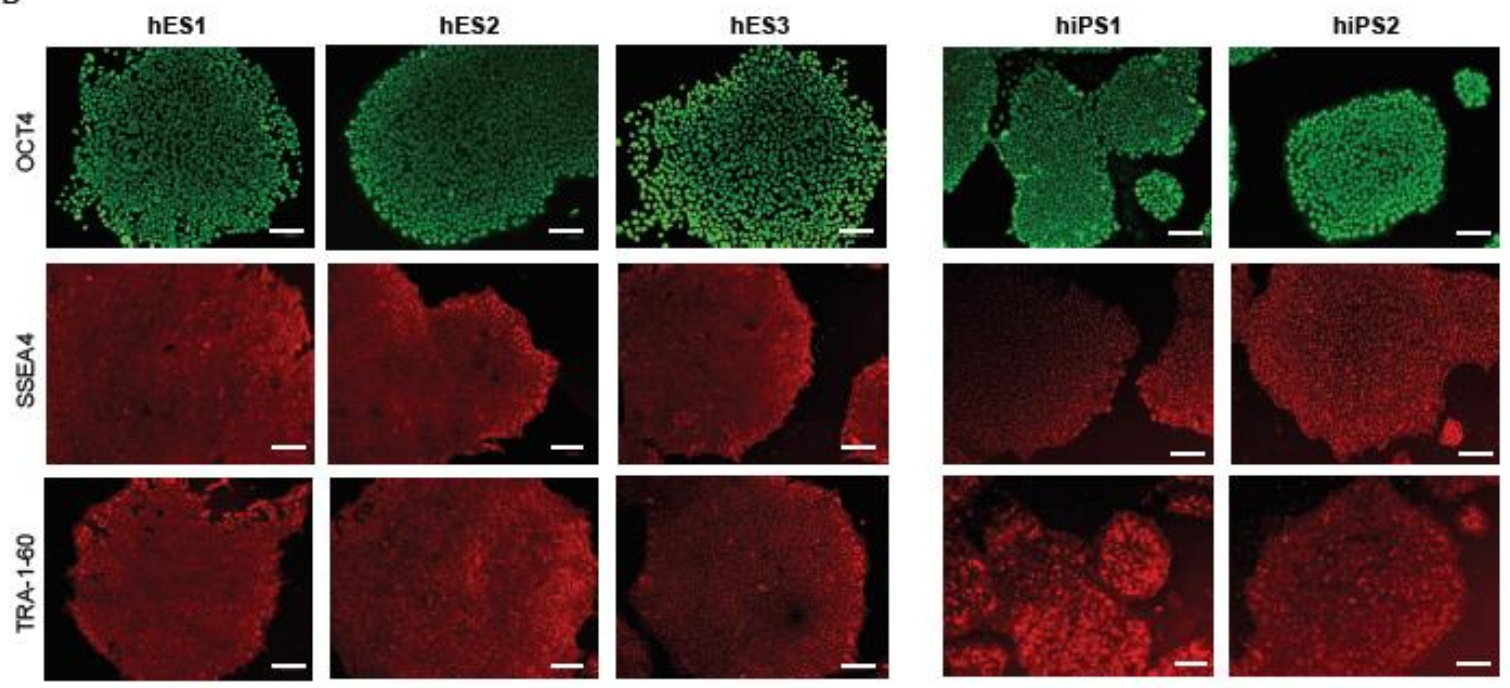

c
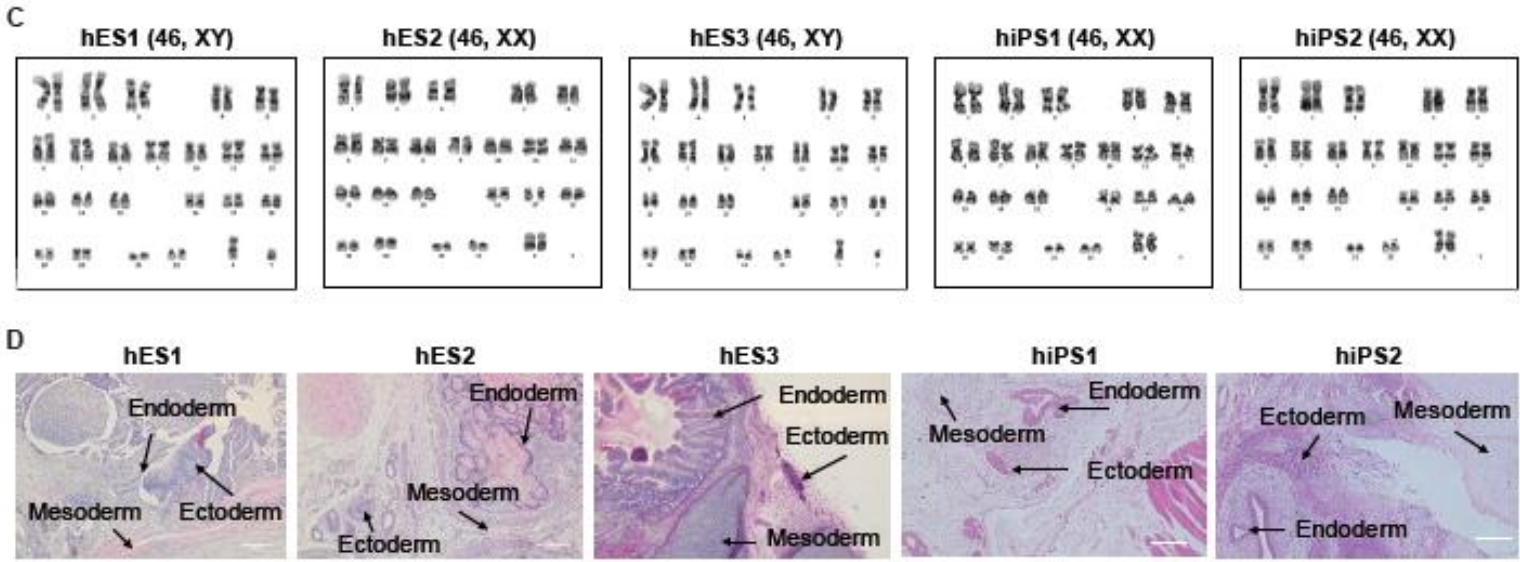

hiPS2

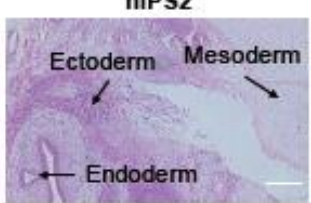

Figure 4. Characterization of hPSCs following fasudil treatment for 2 to 3 months. (A) Quantitative RT-PCR analysis determining OCT4, SOX2, and NANOG expression relative to GAPDH, following the first 24 hours of culture treated with $10 \mu \mathrm{M}$ Y-27632 or fasudil. The difference between the two treatments was not significant. Statistical significance ( $\mathrm{p}$-value) was calculated using one-way ANOVA with Tukey post hoc analysis $(n=9)$. Error bars indicate standard deviations. (B) Immunofluorescence analysis examining OCT4, SSEA-4, and TRA-1-60 expression, following fasudil treatment for 3 months. All 5 lines of PSC expressed the pluripotency marker protein. Scale bars $=100$ $\mu \mathrm{m}$. (C) G-banding karyotype of hPSCs cultured using fasudil over 3 months. Five lines of PSC had no abnormality in the karyotypes. (D) Ectodermal, mesodermal, and endodermal tissue associated with teratoma formation with five lines of hPSCs after 3 months in fasudil-treated culture. In fives lines of PSC, teratomas were well formed, and three germ layers were formed. Scale bars $=200 \mu \mathrm{m}$. 


\subsection{Support of Fasudil During hPSC Differentiation}

We sought to determine whether ROCK2 inhibition could increase the efficiency of hPSC-RPE differentiation. RPE differentiation was carried out using hiPS1 according to the manufacturer's protocol for 14 days[34]. Then, the medium was treated with fasudil or Y-27632 during differentiation. The cells treated with fasudil were in close contact with each other, but cells in the untreated control group were distributed relatively loosely. We also investigated the expression of the RPE marker proteins using flow cytometry. The expression of the RPE marker MITF was significantly higher in the fasudil-treated group (71\%) than in the untreated control group $(42 \%)$ but was similar to that of the Y-27632-treated group (68\%). Another RPE marker, PAX6, was similarly expressed in all three groups (Figure 5A).

Next, to investigate the effect of fasudil on neural crest differentiation, we performed differentiation using hiPS1. After differentiation, the cells of the fasudil group filled the plate, similar to what was observed with the Y-27632-treated cells. However, the cells in the untreated control group were present at low confluency. The expression of neural crest marker proteins was examined by flow cytometry. The efficiency of differentiation among the groups was not significantly different. But, most of the cells in the untreated control group died after neural crest differentiation (Figure 5B). Thus, we confirmed that fasudil increased survivability for neural crest differentiation.

A
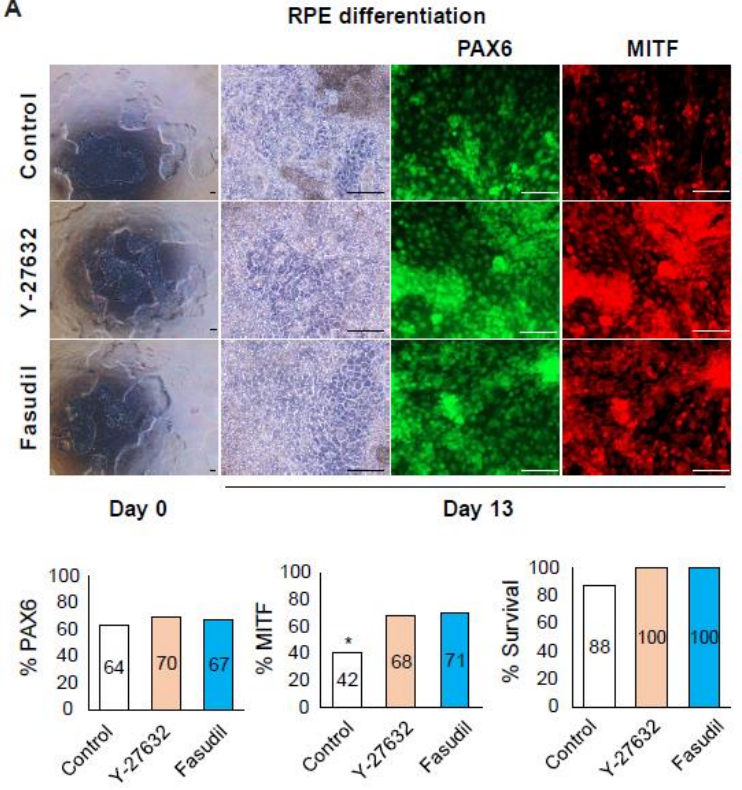

B Neural crest differentiation

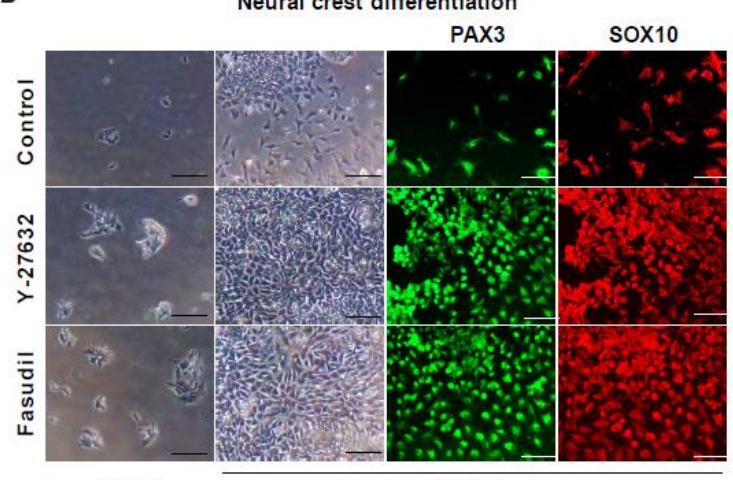

Day 0

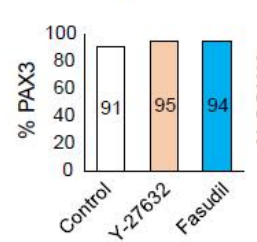

Day 6

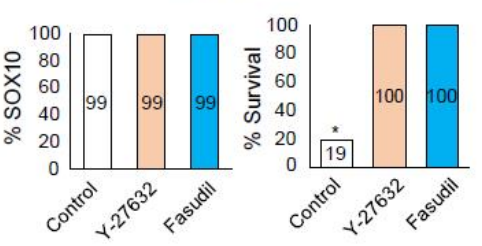

Figure 5. Fasudil promotes the efficiency of differentiation of PSCs. (A) Comparison of the expression of RPE specific markers; PAX6 and MITF by immunocytochemistry (ICC) and flow cytometry at 13 days. The percentage of PAX6 was comparable among groups, while MITF-positive cells in treated groups were higher than untreated. Fasudil did not affect cell survival in RPE differentiation (B) The positive cells of neural crest markers; PAX3 and SOX10 were comparable among groups by ICC and flow cytometry. Fasudil increased cell survival in neural crest differentiation from hiPSCs. Statistical significance ( $\mathrm{p}$-value) was calculated using Fisher's exact test $\left(^{*}: p<0.05\right)$. The experiments were performed with hiPS1.

\subsection{Fasudil can be Used in a Variety of Applications}

To determine whether fasudil could be applied to other PSC studies, for which Y-27632 is already being used, we investigated the effect of fasudil on 3D aggregation and single-cell culture. 
To induce the aggregation of hPSCs for 3D culture, dissociated hES3 cells were cultured for 6 days in suspension $\left(1.2 \times 10^{6}\right.$ cells $/ \mathrm{ml}, 1 \mathrm{ml} / 35 \mathrm{~mm}$ StemFit3D culture dish) using DMEM/F12 medium supplemented with KSR, to which fasudil or Y-27632 was added for the first 24 hours. After 24 hours, hESCs (hES3) began to grow well as floating aggregates, but cells that were untreated with ROCK inhibitors remained as single cells. When fasudil was used, aggregates were formed in almost all molds $(98 \pm 2 \%)$, and there was no significant difference compared with when Y-27632 $(97 \pm 4 \%)$ was used. There was no difference in the size of the aggregates, which were cultured for a total of 6 days using Y-27632 $(138.4 \pm 18.6 \mu \mathrm{m})$ and fasudil $(149.4 \pm 10.2 \mu \mathrm{m})$ (Figure 6A).

To confirm the protective effect of fasudil on the culture of single hPSCs, hES3 cells were dissociated into single cells using Accutase and seeded in a 6-well plate at $5 \times 10^{4}$ per well with $10 \mu \mathrm{M}$ of Y27632 or fasudil. Cells were then counted after 7 days of culture. We observed that treatment with fasudil substantially increased the cell number, relative to the control group, and the fasudil effect was similar to that of Y-27362 (Figure 6B).
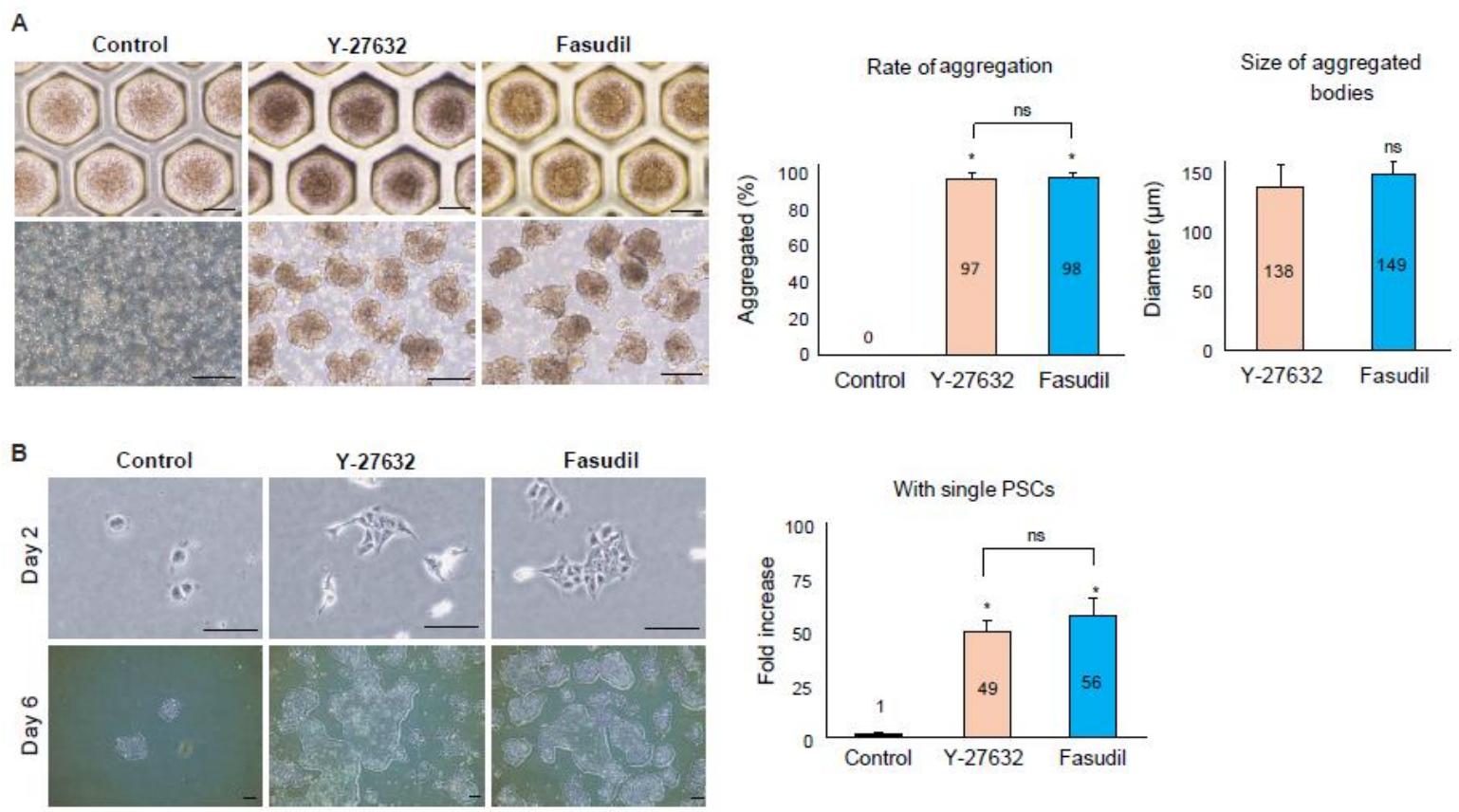

Figure 6. Various applications of fasudil. (A) Supportive effect of fasudil on hES3 survival and growth in suspension culture. Fasudil-treated groups and Y-27632-treated groups were similar in the aggregated cell formation rate and size, but the control group did not form those. Scale bar $=200 \mu \mathrm{m}$ $\left({ }^{*}: p<0.05\right.$; versus control, $\mathrm{n}=14$ ). (B) Seven-day culture of single hES3 cells on vitronectin-coated culture plates in the presence of $10 \mu \mathrm{M}$ Y-27632 or fasudil. Scale Bar $=200 \mu \mathrm{m}$. Also shown, the growth efficiency of single hES3 cells cultured with Y-27632 or fasudil $(*: p<0.05$; versus control, $\mathrm{n}=3$ ). Statistical significance (p-value) was calculated using one-way ANOVA with Tukey post hoc analysis.

Taken together, these results suggested that fasudil could replace Y-27632 and be applied to various stem cell studies. 


\section{Discussion}

ROCK proteins are divided into two subunits, ROCK1 and ROCK2. They play a crucial role in numerous cellular functions, including cell contraction, actin organization, cell migration, and proliferation[49,50]. The roles of the Rho-ROCK-Myosin signaling axis in the formation of the cellcell interactions that are essential for colony formation have been previously demonstrated, and signaling mechanisms are different between multipotent and non-pluripotent cell lines[51]. Therefore, ROCK inhibitors should be used for culturing human pluripotent stem cells (hPSCs); in particular, most researchers use Y-27632 as their preferred ROCK inhibitor.

This study began with a serendipitous question as to why Y-27632 is the primary choice for PSC studies, despite the low cost of fasudil and its ability to improve hPSC survival. This question arose because we are concerned that low-cost, robust, scalable, easy-to-use, and consistent culture methods must be developed to allow widespread applications of hESC/iPSCs for basic research and clinical use. We proposed a PSC culture method using fasudil as a way to overcome this problem. Similar to our findings, several studies have shown that fasudil increases the proliferation of hPSCs; however, in those studies, fasudil was only used as a control group and was not clearly characterized with PSCs through long-term culture.

In an experiment involving ROCK inhibitors, we observed that fasudil itself promoted hPSC survival and proliferation. These observations led to a close examination of the action of fasudil on undifferentiated hPSC cultures. We thought that when PSC was frozen and thawed, simple supplementation with fasudil could significantly improve survival. This effect of fasudil is mediated by the direct inhibition of the ROCK2 intracellular pathway, which is reported to be one of the most important signaling pathways for hPSC survival[52]. Indeed, fasudil dramatically improved cell culturing with hPSC expansion and 3D aggregated suspension. We have demonstrated that this effect occurs because fasudil prevents the dissociated hPSC from cell death. The effect of fasudil seems to be due to the inhibition of ROCK, which promotes depolymerization of actomyosin. Polymerization of actomyosin is essential for cell survival after detachment [53,54]. Furthermore, we reported that the inhibition of ROCK2 by fasudil allowed for long-term culturing of hPSCs through proper gene expression, protein location, function, karyotype maintenance, and metabolism. In particular, fasudil was effective in restoring decreased metabolism after passaging.

We also confirmed that the differentiation of hPSC-RPE increases with fasudil supplementation. Although hPSC-RPE has been developed for the treatment of age-related macular degeneration (AMD), hPSCs must be cultured for several months to obtain a relatively uniform and mature RPE for transplantation. Fasudil supplementation increases RPE differentiation efficiency through the high expression of MITF.

We have shown that fasudil is similar to or more effective than Y-27632 for facilitating cell survival and proliferation. Fasudil can, therefore, replace Y-27632 in PSC studies.

Author Contributions: Conceptualization, E.K. and S.S.; investigation, Y.L. and J.P.; methodology, J.R.D., E.J.S., B.H.L., C.J.K. and S.M.; formal analysis, S.S. and J.Y.L.; validation, S.S., J.C. and S.K.; data curation, S.S., S.J.O. and J.Y.L.; writing-original draft preparation, S.S., E.K., J.H. and J.S.; writing-review and editing, E.K. and S.S.; visualization, S.S.; funding acquisition, E.K.; All authors made comments on the manuscript.

Funding: The study was supported by grants from the National Research Foundation of Korea (NRF2015K1A4A3046807 and 2017M3A9F8031039) and intramural grants (2018, 2019-796) from the Asan Institute for Life Sciences, Asan Medical Center.

Acknowledgments: We thank the Confocal Imaging Core, FACS Core Laboratory, and the Medical Genetics Center at Asan Medical Center for their technical support. We are grateful to the Center for Embryonic Cell and Gene Therapy, Oregon Health \& Science University, for providing hESCs. We also would like to thank the DMPK core facility at the ConveRgence mEDIcine research cenTer (CREDIT), Asan Medical Center, for conducting molecular stability analysis and Patrick Walsh, Vincent Truong, and Caitlin Hill at the Stem Cell 
Institute, University of Minnesota, for transferring expertise in hPSC culture and differentiation. We appreciate to Hyunjeong Kim at Asan Medical Center for performing the ICC and qPCR.

Conflicts of Interest: The authors declare no conflict of interest.

\section{References}

1. McNeish, J. Embryonic stem cells in drug discovery. Nature Reviews Drug Discovery 2004, 3, 70, doi:10.1038/nrd1281.

2. Carpenter, M.K.; Rao, M.S. Concise review: making and using clinically compliant pluripotent stem cell lines. Stem cells translational medicine 2015, 4, 381-388.

3. Ilic, D.; Ogilvie, C. Concise review: Human embryonic stem cells - what have we done? What are we doing? Where are we going? Stem Cells 2017, 35, 17-25.

4. Thomson, J.A.; Itskovitz-Eldor, J.; Shapiro, S.S.; Waknitz, M.A.; Swiergiel, J.J.; Marshall, V.S.; Jones, J.M. Embryonic stem cell lines derived from human blastocysts. science 1998, 282, 1145-1147.

5. Reubinoff, B.E.; Pera, M.F.; Fong, C.-Y.; Trounson, A.; Bongso, A. Embryonic stem cell lines from human blastocysts: somatic differentiation in vitro. Nature biotechnology 2000, 18, 399.

6. $\quad$ Ludwig, T.E.; Levenstein, M.E.; Jones, J.M.; Berggren, W.T.; Mitchen, E.R.; Frane, J.L.; Crandall, L.J.; Daigh, C.A.; Conard, K.R.; Piekarczyk, M.S. Derivation of human embryonic stem cells in defined conditions. Nature biotechnology 2006, 24, 185.

7. Richards, M.; Fong, C.-Y.; Chan, W.-K.; Wong, P.-C.; Bongso, A. Human feeders support prolonged undifferentiated growth of human inner cell masses and embryonic stem cells. Nature biotechnology 2002, $20,933$.

8. Mallon, B.S.; Park, K.-Y.; Chen, K.G.; Hamilton, R.S.; McKay, R.D. Toward xeno-free culture of human embryonic stem cells. The international journal of biochemistry $\mathcal{E}$ cell biology 2006, 38, 1063-1075.

9. Dravid, G.; Ye, Z.; Hammond, H.; Chen, G.; Pyle, A.; Donovan, P.; Yu, X.; Cheng, L. Defining the role of $\mathrm{Wnt} / \beta$-catenin signaling in the survival, proliferation, and self-renewal of human embryonic stem cells. Stem cells 2005, 23, 1489-1501.

10. Steiner, D.; Khaner, H.; Cohen, M.; Even-Ram, S.; Gil, Y.; Itsykson, P.; Turetsky, T.; Idelson, M.; Aizenman, E.; Ram, R. Derivation, propagation and controlled differentiation of human embryonic stem cells in suspension. Nature biotechnology 2010, 28, 361.

11. Bardy, J.a.; Chen, A.K.; Lim, Y.M.; Wu, S.; Wei, S.; Weiping, H.; Chan, K.; Reuveny, S.; Oh, S.K. Microcarrier suspension cultures for high-density expansion and differentiation of human pluripotent stem cells to neural progenitor cells. Tissue Engineering Part C: Methods 2012, 19, 166-180.

12. Villa-Diaz, L.; Ross, A.; Lahann, J.; Krebsbach, P. Concise review: the evolution of human pluripotent stem cell culture: from feeder cells to synthetic coatings. Stem cells 2013, 31, 1-7.

13. Hasegawa, K.; Yasuda, S.-y.; Teo, J.-L.; Nguyen, C.; McMillan, M.; Hsieh, C.-L.; Suemori, H.; Nakatsuji, N.; Yamamoto, M.; Miyabayashi, T. Wnt signaling orchestration with a small molecule DYRK inhibitor provides long-term xeno-free human pluripotent cell expansion. Stem cells translational medicine 2012, 1, 18-28.

14. Watanabe, K.; Ueno, M.; Kamiya, D.; Nishiyama, A.; Matsumura, M.; Wataya, T.; Takahashi, J.B.; Nishikawa, S.; Nishikawa, S.-i.; Muguruma, K. A ROCK inhibitor permits survival of dissociated human embryonic stem cells. Nature biotechnology 2007, 25, 681.

15. Osakada, F.; Jin, Z.-B.; Hirami, Y.; Ikeda, H.; Danjyo, T.; Watanabe, K.; Sasai, Y.; Takahashi, M. In vitro differentiation of retinal cells from human pluripotent stem cells by small-molecule induction. J Cell Sci 2009, 122, 3169-3179. 
16. Huang, S.X.; Islam, M.N.; O'neill, J.; Hu, Z.; Yang, Y.-G.; Chen, Y.-W.; Mumau, M.; Green, M.D.; VunjakNovakovic, G.; Bhattacharya, J. Efficient generation of lung and airway epithelial cells from human pluripotent stem cells. Nature biotechnology 2014, 32, 84 .

17. Rezania, A.; Bruin, J.E.; Arora, P.; Rubin, A.; Batushansky, I.; Asadi, A.; O'dwyer, S.; Quiskamp, N.; Mojibian, M.; Albrecht, T. Reversal of diabetes with insulin-producing cells derived in vitro from human pluripotent stem cells. Nature biotechnology 2014, 32, 1121.

18. Braam, S.R.; Tertoolen, L.; van de Stolpe, A.; Meyer, T.; Passier, R.; Mummery, C.L. Prediction of druginduced cardiotoxicity using human embryonic stem cell-derived cardiomyocytes. Stem cell research 2010, 4, 107-116.

19. Higuchi, A.; Kumar, S.S.; Ling, Q.-D.; Alarfaj, A.A.; Munusamy, M.A.; Murugan, K.; Hsu, S.-T.; Benelli, G.; Umezawa, A. Polymeric design of cell culture materials that guide the differentiation of human pluripotent stem cells. Progress in Polymer Science 2017, 65, 83-126.

20. Sindeeva, O.A.; Verkhovskii, R.A.; Sarimollaoglu, M.; Afanaseva, G.A.; Fedonnikov, A.S.; Osintsev, E.Y.; Kurochkina, E.N.; Gorin, D.A.; Deyev, S.M.; Zharov, V.P. New Frontiers in Diagnosis and Therapy of Circulating Tumor Markers in Cerebrospinal Fluid In Vitro and In Vivo. Cells 2019, 8, 1195.

21. Paleček, J.; Zweigerdt, R.; Olmer, R.; Martin, U.; Kirschning, A.; Dräger, G. A practical synthesis of RhoKinase inhibitor Y-27632 and fluoro derivatives and their evaluation in human pluripotent stem cells. Organic E biomolecular chemistry 2011, 9, 5503-5510.

22. Loge, C.; Siomboing, X.; Wallez, V.; Scalbert, E.; Bennejean, C.; Cario-Tourmaniantz, C.; Loirand, G.; Gressier, B.; Pacaud, P.; Luyckx, M. Synthesis and pharmacological study of rho-kinase inhibitors: Pharmacomodulations on the lead compound Fasudil. Journal of enzyme inhibition and medicinal chemistry 2003, 18, 127-138.

23. Braam, S.R.; Zeinstra, L.; Litjens, S.; Ward-van Oostwaard, D.; van den Brink, S.; van Laake, L.; Lebrin, F.; Kats, P.; Hochstenbach, R.; Passier, R. Recombinant vitronectin is a functionally defined substrate that supports human embryonic stem cell self-renewal via $\alpha \mathrm{V} \beta 5$ integrin. Stem cells 2008, 26, 2257-2265.

24. Ma, H.; Morey, R.; O'Neil, R.C.; He, Y.; Daughtry, B.; Schultz, M.D.; Hariharan, M.; Nery, J.R.; Castanon, R.; Sabatini, K. Abnormalities in human pluripotent cells due to reprogramming mechanisms. Nature 2014, 511, 177.

25. Nie, Y.; Walsh, P.; Clarke, D.L.; Rowley, J.A.; Fellner, T. Scalable passaging of adherent human pluripotent stem cells. PloS one 2014, 9, e88012.

26. Reubinoff, B.; Pera, M.; Vajta, G.; Trounson, A. Effective cryopreservation of human embryonic stem cells by the open pulled straw vitrification method. Human reproduction 2001, 16, 2187-2194.

27. Yamaguchi, H.; Kasa, M.; Amano, M.; Kaibuchi, K.; Hakoshima, T. Molecular mechanism for the regulation of rho-kinase by dimerization and its inhibition by fasudil. Structure 2006, 14, 589-600.

28. Motevaseli, E.; Shirzad, M.; Akrami, S.M.; Mousavi, A.-S.; Mirsalehian, A.; Modarressi, M.H. Normal and tumour cervical cells respond differently to vaginal lactobacilli, independent of $\mathrm{pH}$ and lactate. Journal of medical microbiology 2013, 62, 1065-1072.

29. Aryal, P.; Kim, K.; Park, P.H.; Ham, S.; Cho, J.; Song, K. Baicalein induces autophagic cell death through AMPK/ULK1 activation and downregulation of $\mathrm{m}$ TORC 1 complex components in human cancer cells. The FEBS journal 2014, 281, 4644-4658.

30. Wang, B.; Niu, D.; Lai, L.; Ren, E.C. p53 increases MHC class I expression by upregulating the endoplasmic reticulum aminopeptidase ERAP1. Nature communications 2013, 4, 2359. 
31. Shultz, L.D.; Ishikawa, F.; Greiner, D.L. Humanized mice in translational biomedical research. Nature Reviews Immunology 2007, 7, 118.

32. Ruifrok, A.C.; Johnston, D.A. Quantification of histochemical staining by color deconvolution. Analytical and quantitative cytology and histology 2001, 23, 291-299.

33. Ji, Y.; Hao, H.; Reynolds, K.; McMahon, M.; Zhou, C.J. Wnt Signaling in Neural Crest Ontogenesis and Oncogenesis. Cells 2019, 8, 1173.

34. Geng, Z.; Walsh, P.J.; Truong, V.; Hill, C.; Ebeling, M.; Kapphahn, R.J.; Montezuma, S.R.; Yuan, C.; Roehrich, H.; Ferrington, D.A. Generation of retinal pigmented epithelium from iPSCs derived from the conjunctiva of donors with and without age related macular degeneration. PloS one 2017, 12, $\mathrm{e} 0173575$.

35. Frith, T.J.; Tsakiridis, A. Efficient Generation of Trunk Neural Crest and Sympathetic Neurons from Human Pluripotent Stem Cells Via a Neuromesodermal Axial Progenitor Intermediate. Current protocols in stem cell biology 2019, e81.

36. Ding, J.; Yu, J.-Z.; Li, Q.-Y.; Wang, X.; Lu, C.-Z.; Xiao, B.-G. Rho kinase inhibitor Fasudil induces neuroprotection and neurogenesis partially through astrocyte-derived G-CSF. Brain, behavior, and immunity 2009, 23, 1083-1088.

37. Breitenlechner, C.; Gaßel, M.; Hidaka, H.; Kinzel, V.; Huber, R.; Engh, R.A.; Bossemeyer, D. Protein kinase A in complex with Rho-kinase inhibitors Y-27632, Fasudil, and H-1152P: structural basis of selectivity. Structure 2003, 11, 1595-1607.

38. Zhang, L.; Xu, Y.; Xu, J.; Wei, Y.; Xu, X. Protein kinase A inhibitor, H89, enhances survival and clonogenicity of dissociated human embryonic stem cells through Rho-associated coiled-coil containing protein kinase (ROCK) inhibition. Human reproduction 2016, 31, 832-843.

39. Kinehara, M.; Kawamura, S.; Tateyama, D.; Suga, M.; Matsumura, H.; Mimura, S.; Hirayama, N.; Hirata, M.; Uchio-Yamada, K.; Kohara, A. Protein kinase C regulates human pluripotent stem cell self-renewal. PloS one 2013, 8, e54122.

40. Uehata, M.; Ishizaki, T.; Satoh, H.; Ono, T.; Kawahara, T.; Morishita, T.; Tamakawa, H.; Yamagami, K.; Inui, J.; Maekawa, M. Calcium sensitization of smooth muscle mediated by a Rho-associated protein kinase in hypertension. Nature 1997, 389, 990.

41. Chen, M.; Liu, A.; Ouyang, Y.; Huang, Y.; Chao, X.; Pi, R. Fasudil and its analogs: a new powerful weapon in the long war against central nervous system disorders? Expert opinion on investigational drugs 2013, 22, 537-550.

42. Strelow, J.; Dewe, W.; Iversen, P.W.; Brooks, H.B.; Radding, J.A.; McGee, J.; Weidner, J. Mechanism of action assays for enzymes. In Assay Guidance Manual [Internet], Eli Lilly \& Company and the National Center for Advancing Translational Sciences: 2012.

43. Vernardis, S.I.; Terzoudis, K.; Panoskaltsis, N.; Mantalaris, A. Human embryonic and induced pluripotent stem cells maintain phenotype but alter their metabolism after exposure to ROCK inhibitor. Scientific reports 2017, 7, 42138 .

44. Dibble, C.C.; Manning, B.D. Signal integration by mTORC1 coordinates nutrient input with biosynthetic output. Nature cell biology 2013, 15, 555.

45. Jones, R.G.; Plas, D.R.; Kubek, S.; Buzzai, M.; Mu, J.; Xu, Y.; Birnbaum, M.J.; Thompson, C.B. AMPactivated protein kinase induces a p53-dependent metabolic checkpoint. Molecular cell 2005, 18, 283-293.

46. Wei, X.; Luo, L.; Chen, J. Roles of mTOR Signaling in Tissue Regeneration. Cells 2019, 8, 1075. 
47. Garziera, M.; Cecchin, E.; Giorda, G.; Sorio, R.; Scalone, S.; Mattia, E.D.; Roncato, R.; Gagno, S.; Poletto, E.; Romanato, L. Clonal Evolution of TP53 c. 375+ 1G> A Mutation in Pre-and Post-Neo-Adjuvant Chemotherapy (NACT) Tumor Samples in High-Grade Serous Ovarian Cancer (HGSOC). Cells 2019, 8, 1186.

48. Nichols, J.; Zevnik, B.; Anastassiadis, K.; Niwa, H.; Klewe-Nebenius, D.; Chambers, I.; Schöler, H.; Smith, A. Formation of pluripotent stem cells in the mammalian embryo depends on the POU transcription factor Oct4. Cell 1998, 95, 379-391.

49. Amano, M.; Nakayama, M.; Kaibuchi, K. Rho-kinase/ROCK: a key regulator of the cytoskeleton and cell polarity. Cytoskeleton 2010, 67, 545-554.

50. Totsukawa, G.; Wu, Y.; Sasaki, Y.; Hartshorne, D.J.; Yamakita, Y.; Yamashiro, S.; Matsumura, F. Distinct roles of MLCK and ROCK in the regulation of membrane protrusions and focal adhesion dynamics during cell migration of fibroblasts. The Journal of cell biology 2004, 164, 427-439.

51. Harb, N.; Archer, T.K.; Sato, N. The Rho-Rock-Myosin signaling axis determines cell-cell integrity of self-renewing pluripotent stem cells. PloS one 2008, 3, e3001.

52. Meng, Y.; Ren, Z.; Xu, F.; Zhou, X.; Song, C.; Wang, V.Y.-F.; Liu, W.; Lu, L.; Thomson, J.A.; Chen, G. Nicotinamide Promotes Cell Survival and Differentiation as Kinase Inhibitor in Human Pluripotent Stem Cells. Stem cell reports 2018, 11, 1347-1356.

53. Busche, S.; Descot, A.; Julien, S.; Genth, H.; Posern, G. Epithelial cell-cell contacts regulate SRF-mediated transcription via Rac-actin-MAL signalling. Journal of cell science 2008, 121, 1025-1035.

54. Connell, L.E.; Helfman, D.M. Myosin light chain kinase plays a role in the regulation of epithelial cell survival. Journal of cell science 2006, 119, 2269-2281. 